\title{
TEM Sample Preparation of Buried Interfaces in Porous Layered Materials
}

Warren L. York ${ }^{1}$, Dong Ding ${ }^{2}$, Hanping Ding ${ }^{2}$ Joshua D. Sugar $^{1}$

${ }^{1}$ Sandia National Laboratories, Livermore, California, United States, ${ }^{2}$ Idaho National Laboratory, Idaho Falls, Idaho, United States

* Corresponding author: wlyork@ sandia.gov

Focused Ion Beam (FIB) is a widely used technique in preparing traditional lamella type Transmission Electron Microscopy (TEM) samples with a cross sectional geometry [1]. Usually, samples are extracted from a specimen where the region of interest is within a few microns of the top surface of the sample. However, additional challenges exist when preparing a lamella where the area of interest for TEM observations is $>10 \mu \mathrm{m}$ from the top surface. For one, the ion beam does not have a large enough depth of focus to remain in focus for the entirety of its interaction with a large ( >10 $\mu \mathrm{m}$ long) sample (Fig 1a). Targeting a region to thin with an out of focus ion beam is not ideal. Furthermore, when the region of interest is buried below porous material, curtaining from the pores will cause uneven thinning and prevent the fabrication of a uniformly thin sample. Presently, there are several TEM preparation techniques described in the literature, for example reference [2] that discuss inversion and a rocking polish to reduce curtaining artifacts. Here, we demonstrate an example of how to mount and thin a sample for TEM and EELS analysis when the area of interest is in the middle of a multi-layered stack of porous materials.

The sample chosen was an HTE (High Temperature Electrolysis) solid oxide fuel cell structure that is used to split water to make hydrogen fuel. The layers were a La-Sr-Co-Fe oxide electrode/Gd-doped ceria buffer layer/yttria-stabilized zirconia electrolyte/Ni-yttria-stabilized zirconia electrode (Fig 1a). The area of interest for TEM analysis was the interface between the buffer layer and the electrolyte, which had layers of porous electrode materials on either side, each $\sim 40 \mu \mathrm{m}$ thick. Milling into porous material can be difficult due to curtaining and uneven milling, which can result in voids and contrast-causing artifacts in the final thinned area. The first step was to prepare a cross section in a metallographic mount that revealed the full layered structure of the fuel cell. A lamella containing the area of interest was then removed from the cross-section mount. The lamella was then rotated about the liftout needle $180^{\circ}$, which is accompanied by a $90^{\circ}$ in plane rotation, so the lamella was orientated with the less porous electrolyte layer at the top. Having the nonporous, solid, and dense layer at the top helps prevent curtain formation and assists in minimizing the formation of voids in the area of interest. The top electrolyte layer was still too thick for easy thinning of the interface of interest. So, before starting the thinning steps some of the top layer was removed by placing the sample on a $38^{\circ}$ pretilt and $14^{\circ}$ stage tilt to make a perpendicular cut with the ion beam. The removal was stopped when a few microns of the electrolyte YSZ layer remained such that the sample had the usual geometry for a TEM liftout with the region of interest a few microns below the top sample surface. Next, a protective layer of Pt was placed on the top surface. The lamella was then prepared using the usual recipe for thinning at $30 \mathrm{kV}$ with the usual current and window size combinations. The sample was then thinned down to the appropriate (electron transparent) thickness for TEM and EELS analysis using the $5 \mathrm{kV}$ beam on the FIB.

The resulting thin sample is shown in figure 1d. The area of interest was thin enough for TEM and EELS analysis. A $300 \mathrm{kV}$ STEM image showing the area of interest is shown in Figure 2a/b where the grain structure of the area of interest is visible. This technique provides high-quality TEM results and demonstrates that some traditional metallography processes combined with reorienting a sample several times in the FIB before final thinning is needed to get a high-quality result in the region of interest [3]. 
a.

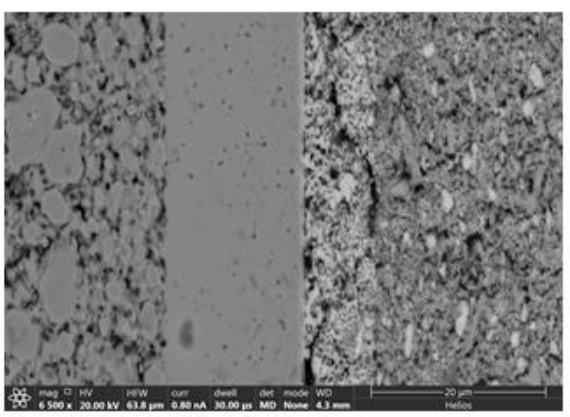

.

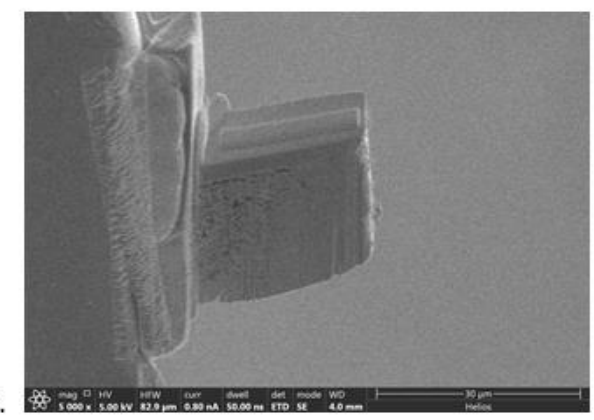

b.

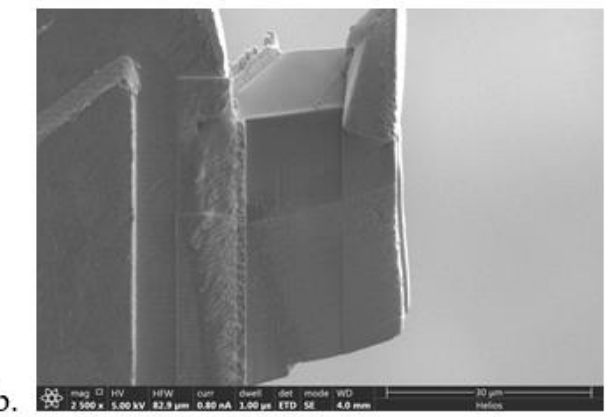

d.

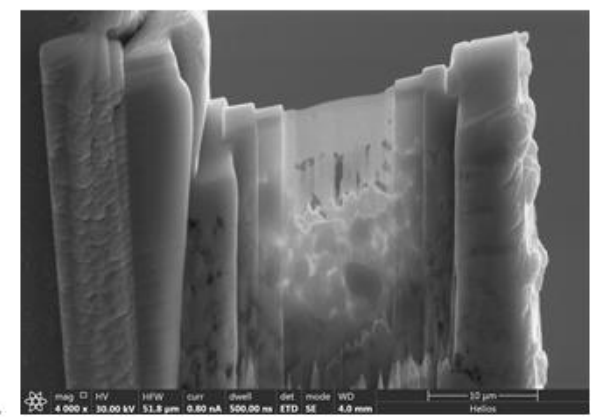

Figure 1. (a) SEM of cross section (right to left LSCF oxide electrode/Gd-doped ceria buffer layer/yttriastabilized zirconia electrolyte/Ni-yttria-stabilized zirconia electrode). (b) SEM image sample on post before cleanup of top. (c) SEM image sample after cleanup. (d) Final thin sample ready for TEM.

a.

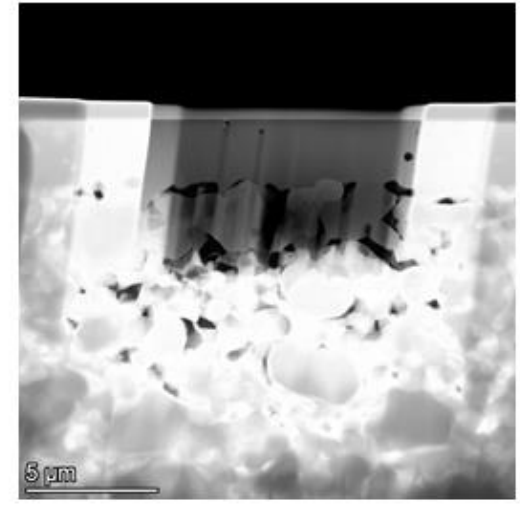

b.

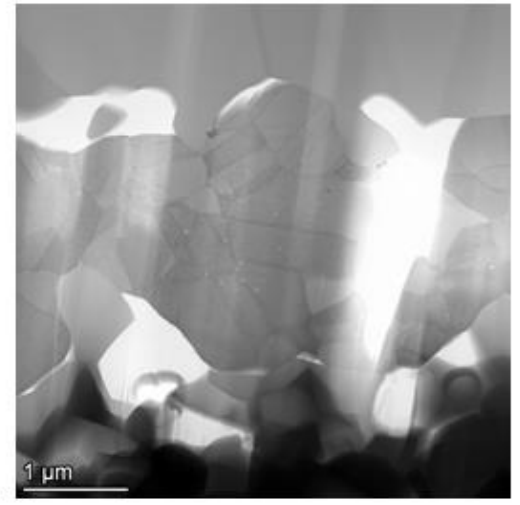

Figure 2. (a) BF TEM image of thinned particle. (b) HAADF TEM image of thinned area. EM BF image of thinned area.

\section{References}

[1] L.A. Giannuzzi and F.A. Stevie, Micron volume 30 issue 3 (1999), p. 197-204.

[2] Andrey Denisyuk, Tomas Hrncir, Fozef Obona, Sharang, Martin Petrenec and Jan Michalicka, Microscopy and Microanalysis Volume 23 Issue 3 (2017) p. 484 - 490

[3] Sandia National Laboratories is a multimission laboratory managed and operated by National Technology and Engineering Solutions of Sandia LLC, a wholly owned subsidiary of Honeywell International Inc., for the U.S. Department of Energy's National Nuclear Security Administration under contract DE-NA0003525. This paper describes objective technical results and analysis. Any subjective views or opinions that might be expressed in the paper do not necessarily represent the views of the U.S. Department of Energy or the United States Government. 